Article

\title{
Fractional Supersymmetric Hermite Polynomials
}

\author{
Fethi Bouzeffour ${ }^{1, *(1)}$ and Wissem Jedidi ${ }^{2,3}$ (1) \\ 1 Department of Mathematics, College of Sciences, King Saud University, P. O. Box 2455, \\ Riyadh 11451, Saudi Arabia \\ 2 Department of Statistics \& OR, King Saud University, P.O. Box 2455, Riyadh 11451, Saudi Arabia; \\ wissem.jedidi@fst.utm.tn \\ 3 Faculté des Sciences de Tunis, LR11ES11 Laboratoire d'Analyse Mathématiques et Applications, \\ Université de Tunis El Manar, Tunis 2092, Tunisia \\ * Correspondence: fbouzaffour@ksu.edu.sa
}

Received: 12 December 2019; Accepted: 31 January 2020; Published: 5 February 2020

\begin{abstract}
We provide a realization of fractional supersymmetry quantum mechanics of order $r$, where the Hamiltonian and the supercharges involve the fractional Dunkl transform as a Klein type operator. We construct several classes of functions satisfying certain orthogonality relations. These functions can be expressed in terms of the associated Laguerre orthogonal polynomials and have shown that their zeros are the eigenvalues of the Hermitian supercharge. We call them the supersymmetric generalized Hermite polynomials.
\end{abstract}

Keywords: orthogonal polynomials; difference-differential operator; supersymmetry

\section{Introduction}

Supersymmetry relates bosons and fermions on the basis of $\mathbb{Z}_{2}$-graded superalgebras [1,2], where the fermionic set is realized in terms of matrices of finite dimension or in terms of Grassmann variables [3]. The supersymmetric quantum mechanics (SUSYQM), introduced by Witten [2], may be generated by three operators $Q_{-}, Q_{+}$and $H$ satisfying

$$
Q_{ \pm}^{2}=0, \quad\left[Q_{ \pm}, H\right]=0, \quad\left\{Q_{-}, Q_{+}\right\}=H .
$$

Superalgebra (1) corresponds to the case $N=2$ supersymmetry. The usual construction of Witten's supersymmetric quantum mechanics with the superalgebra (1) is performed by introduction of fermion degrees of freedom (realized in a matrix form, or in terms of Grassmann variables) which commute with bosonic degrees of freedom. Another realization of supersymmetric quantum mechanics, called minimally bosonized supersymmetric quantum $[1,4,5]$, is built by taking the supercharge as the following Dunkl-type operator:

$$
Q=\partial_{x} R+v(x)
$$

where $v(x)$ is a superpotential.

The fractional supersymmetric quantum mechanics of order $r$ (FSUYQM) are an extension of the ordinary supersymmetric quantum mechanics for which the $\mathbb{Z}_{2}$-graded superalgebras are replaced by a $\mathbb{Z}_{r}$-graded superalgberas $[3,6,7]$. The framework of the fractional supersymmetric quantum mechanics has been shown to be quite fruitful. Amongst many works, we may quote the deformed Heisenberg algebra introduced in connection with parafermionic and parabosonic systems [3,4], the $C_{\lambda}$-extended oscillator algebra developed in the framework of parasupersymmetric quantum mechanics [8], and the generalized Weyl-Heisenberg algebra $W_{k}$ related to $\mathbb{Z}_{k}$-graded supersymmetric quantum mechanics [3]. 
Note that the construction of fractional supersymmetric quantum mechanics without employment of fermions and parafermions degrees of freedom was started in $[4,9,10]$. In particular, the idea of realization of fractional supersymmetry in the form as it was presented in $[3,8]$ was initially proposed in [4] and also in [9]. In this work, we develop a fractional supersymmetric quantum of order $r$ without parafermonic degrees of freedom. We essentially use a difference-differential operators generated from a special case of the well known fractional Dunkl transform. We then investigate the characteristics of the $(r)$-scheme.

The paper is organized as follows. In Section 2, we discuss some of basic properties of the fractional Dunkl transform and we define the generalized Klein operator. In Section 3, we present a realization of the fractional supersymmetric quantum mechanics and we construct a basis involving the generalized Hermite functions that diagonalize the Hamiltonian. In Section 4, we define the associated generalized Hermite polynomials and we provide its weight function and we show that the eigenvalues of the supercharge are the zeros of the associated generalized Hermite polynomials.

\section{Preliminaries}

Recall that the fractional Dunkl transform on the real line, introduced in [11,12], is both an extension of the fractional Hankel transform and the Fourier transform. For $0<|\alpha|<\pi$, the fractional Dunkl transform is defined by:

$$
\mathcal{F}_{v}^{\alpha} f(t)=\frac{e^{i(v+1 / 2)(\tilde{\alpha} \pi / 2-\alpha)}}{(2|\sin (\alpha)|)^{v+1 / 2} \Gamma(v+1 / 2)} \int_{\mathbb{R}} e^{-i \frac{t^{2}+x^{2}}{\tan \alpha}} \mathcal{E}_{v}\left(\frac{i t x}{\sin \alpha}\right) f(x)|x|^{2 v} d x,
$$

where

$$
\tilde{\alpha}=\operatorname{sgn}(\sin (\alpha))
$$

and

$$
\begin{aligned}
\mathcal{E}_{v}(x) & :=\mathcal{J}_{v-1 / 2}(i x)+\frac{x}{2 v+1} \mathcal{J}_{v+1 / 2}(i x), \\
\mathcal{J}_{v}(x) & :=\Gamma(v+1)(2 / x)^{v} J_{v}(x) .
\end{aligned}
$$

Notice that $J_{v}(x)$ is the standard Bessel function ([13] Ch. 10) and $\Gamma(x)$ is the Gamma function. It is well known that, for $v>0$, the function $\mathcal{E}_{v}(\lambda x)$ is the unique analytic solution of the following system that can be found in [14]:

$$
\left\{\begin{array}{l}
Y_{v} \mathcal{E}_{v}(\lambda x)=i \lambda \mathcal{E}_{v}(\lambda x), \\
\mathcal{E}_{v}(0)=1
\end{array}\right.
$$

where $Y_{v}$ is the Dunkl operator related to root system $A_{1}$ (see ([14] Definition 4.4.2))), which is a differential-difference operator, depending on a parameter $v \in \mathbb{R}$ and acting on $C^{\infty}(\mathbb{R})$ as:

$$
Y_{v}:=\frac{d}{d x}+\frac{v}{x}(1-R)
$$

where $R$ is the Klein operator :

$$
(R f)(x)=f(-x)
$$


The operator $Y_{v}$ is also related by a simple similarity transformation to the Yang-Dunkl operator used in Refs. $[1,4,10]$. The corresponding Dunkl harmonic oscillator and the annihilation and creation operators take the forms [15]

$$
\begin{aligned}
& H_{v}=-\frac{1}{2} Y_{v}^{2}+\frac{1}{2} x^{2}=-\frac{1}{2} \frac{d^{2}}{d x^{2}}-\frac{v}{x} \frac{d}{d x}+\frac{v}{2 x^{2}}(1-R)+\frac{1}{2} x^{2}, \\
& A_{-}=\frac{1}{\sqrt{2}}\left(Y_{v}+x\right), \quad A_{+}=\frac{1}{\sqrt{2}}\left(-Y_{v}+x\right) .
\end{aligned}
$$

They satisfy the (anti)commutation relations

$$
\left[A_{-}, A_{+}\right]=1+2 v R, \quad R^{2}=1, \quad\left\{A_{ \pm}, R\right\}=0, \quad\left[1, A_{ \pm}\right]=[1, R]=0 .
$$

The generators $1, A_{ \pm}, R$, and relations (7) give us a realization of the $R$-deformed Heisenberg algebra $[1,10]$. In $[9,13]$, the authors show that the $R$-deformed algebra is intimately related to parabosons, parafermions [13] and to the $\operatorname{os} p(1 \mid 2) \cos p(2 \mid 2)$ superalgebras.

From now, we assume that $v>0$. The adjoint $Y_{v}^{*}$ of the Dunkl operators $Y_{v}$ with domain $\mathcal{S}(\mathbb{R})$ (the space $\mathcal{S}(\mathbb{R})$ being dense in $L^{2}\left(\mathbb{R},|x|^{2 v} d x\right)$ ) is $-Y_{v}$ and therefore the operator $H_{v}$ is self-adjoint, its spectrum is discrete, and the wave functions corresponding to the well-known eigenvalues

$$
\lambda_{n}=n+v+\frac{1}{2}, \quad n=0,1,2, \cdots
$$

are given by

$$
\psi_{n}^{(v)}(x)=\gamma_{n}^{-1 / 2} e^{-x^{2} / 2} H_{n}^{(v)}(x)
$$

where

$$
\gamma_{n}=2^{2 n} \Gamma\left(\left[\frac{n}{2}\right]+1\right) \Gamma\left(\left[\frac{n+1}{2}\right]+v+\frac{1}{2}\right), n=0,1,2, \cdots .
$$

$[x]$ denotes the greatest integer function and $H_{n}^{(v)}(x)$ is the generalized Hermite polynomial introduced by Szegö [15-17] and obtained from Laguerre polynomial $L_{n}^{(v)}(x)$ as follows:

$$
\left\{\begin{array}{l}
H_{2 n}^{(v)}(x)=(-1)^{n} 2^{2 n} n ! L_{n}^{\left(v-\frac{1}{2}\right)}\left(x^{2}\right) \\
H_{2 n+1}^{(v)}(x)=(-1)^{n} 2^{2 n+1} n ! x L_{n}^{\left(v+\frac{1}{2}\right)}\left(x^{2}\right) .
\end{array}\right.
$$

It is well known that for $v>0$, these polynomials satisfy the orthogonality relations :

$$
\int_{\mathbb{R}} H_{n}^{(v)}(x) H_{m}^{(v)}(x)|x|^{2 v} e^{-x^{2}} d x=\gamma_{n} \delta_{n m} .
$$

We define the generalized Klein operator $K$ as a special case of the fractional Dunkl transform $\mathcal{F}_{v}^{\alpha}$ corresponding to $\alpha=\frac{2 \pi}{r}$. That is,

$$
K=\mathcal{F}_{v}^{\frac{2 \pi}{r}}
$$

It is well known that the wave functions $\psi_{n}^{(v)}(x)$ form an orthonormal basis of $L^{2}\left(\mathbb{R},|x|^{2 v} d x\right)$ and are also eigenfunctions of the Fourier-Dunkl transform $[11,12,15]$. In particular, the generalized Klein operator $K$ acts on the wave functions $\psi_{n}^{(v)}(x)$ as:

$$
K \psi_{n}(x)=\varepsilon_{r}^{n} \psi_{n}^{(v)}(x), \quad \varepsilon_{r}=e^{\frac{2 i \pi}{r}} .
$$


Let us consider the $\mathbb{Z}_{r}$-grading structure on the space $L^{2}\left(\mathbb{R},|x|^{2 v} d x\right)$ as

$$
L^{2}\left(\mathbb{R},|x|^{2 v} d x\right)=\bigoplus_{j=0}^{r-1} L_{j}^{2}\left(\mathbb{R},|x|^{2 v} d x\right)
$$

where $L_{j}^{2}\left(\mathbb{R},|x|^{2 v} d x\right)$ is a linear subspace of $L^{2}\left(\mathbb{R},|x|^{2 v} d x\right)$ generated by the generalized wave functions

$$
\left\{\psi_{n r+j}^{(v)}(x): n=0,1,2, \cdots\right\} .
$$

For $j=0,1, \cdots, r-1$, we denote by $\Pi_{j}$, the orthogonal projection from $L^{2}\left(\mathbb{R},|x|^{2 v} d x\right)$ onto its subspace $L_{j}^{2}\left(\mathbb{R},|x|^{2 v} d x\right)$. The action of $\Pi_{j}$ on $L^{2}\left(\mathbb{R},|x|^{2 v} d x\right)$ can be taken to be

$$
\Pi_{k} \psi_{n r+j}^{(v)}(x)=\delta_{k j} \psi_{n r+j}^{(v)}(x) .
$$

It is clear that they form a system of resolution of the identity:

$$
\Pi_{0}+\Pi_{1}+\cdots+\Pi_{r-1}=1, \quad \Pi_{i} \Pi_{j}=\delta_{i j} \Pi_{i}, \quad \Pi_{j}^{*}=\Pi_{j} .
$$

Note that the orthogonal projection $\Pi_{j}$ is related to the Klein operator $K$ by

$$
\Pi_{j}=\frac{1}{r} \sum_{l=0}^{r-1} \varepsilon_{r}^{-l j} K^{l}
$$

\section{Fractional Supersymmetric Dunkl Harmonic Oscillator}

In this section, we shall present a construction of the fractional supersymmetric quantum mechanics of order $r(r=2,3, \ldots)$ by using the generalized Klein's operator defined in Equation (11). Following Khare [6,7], a fractional supersymmetric quantum mechanics model of arbitrary order $r$ can be developed by generalizing the fundamental Equations (1) to the forms

$$
\begin{aligned}
& Q_{ \pm}^{r}=0, \quad\left[H, Q_{ \pm}\right]=0, \quad Q_{-}^{+}=Q_{+} \\
& Q_{-}^{r-2} H=Q_{-}^{r-1} Q_{+}+Q_{-}^{r-2} Q_{+} Q_{-}+\cdots+Q_{-} Q_{+} Q_{-}^{r-2}+Q_{+} Q_{-}^{r-1} .
\end{aligned}
$$

We introduce the supercharges $Q_{-}$and $Q_{+}$as :

$$
Q_{-}=\frac{1}{\sqrt{2}}\left(Y_{v}+x\right)\left(1-\Pi_{0}\right), \quad Q_{+}=\frac{1}{\sqrt{2}}\left(1-\Pi_{0}\right)\left(-Y_{v}+x\right)
$$

and the fractional supersymmetric Dunkl harmonic oscillator $\mathcal{H}_{v}$ by

$$
\mathcal{H}_{v}=-(r-1) \frac{1}{2} Y_{v}^{2}+(r-1) \frac{1}{2} x^{2}-\sum_{k=0}^{r-1} \Theta_{k} \Pi_{r-k-1},
$$

where

$$
\Theta_{k}=\frac{(r-1)(r-2 k)}{2}+2 v\left[\frac{2 r+(-1)^{k}-1}{4}\right] R, \quad k=0, \cdots, r-1,
$$

and recall that [.] denotes the greatest integer function. Obviously, the operators $Q_{ \pm}$and $\mathcal{H}_{v}$ with common domain $\mathcal{S}(\mathbb{R})$ are densely defined in the Hilbert space $L^{2}\left(\mathbb{R},|x|^{2 v} d x\right)$ and have the Hermitian conjugation relations

$$
\mathcal{H}_{v}^{*}=\mathcal{H}_{v}, \quad Q_{-}^{*}=Q_{+} .
$$


Furthermore, they satisfy the intertwining relations valid for $s=0, \cdots, r-1$ :

$$
\Pi_{s} Q_{-}=Q_{-} \Pi_{s+1}, \quad Q_{+} \Pi_{s}=\Pi_{s+1} Q_{+}, \quad \mathcal{H}_{v} \Pi_{s}=\Pi_{s} \mathcal{H}_{v}
$$

Proposition 1. The supercharges $Q_{ \pm}$are nilpotent operators of order $r$.

Proof. By making use of the following relations

$$
Y_{v} \Pi_{s}=\Pi_{s-1} Y_{v}, \quad x \Pi_{s}=\Pi_{s+1} x
$$

we can easily show by induction that

$$
Q_{-}^{k}= \begin{cases}A_{-}^{k}\left(1-\sum_{s=0}^{k-1} \Pi_{s}\right), & \text { if } 1 \leq k \leq r-1 \\ 0, & \text { if } k=r\end{cases}
$$

Since $Q_{+}=Q_{-}^{*}$, we also have $Q_{+}^{r}=0$.

The first main result is

Theorem 1. The Hermitian operators $Q_{-}, Q_{+}$and $\mathcal{H}_{v}$ defined in Equations (14) and (15) satisfy the commutation relations:

$$
\begin{aligned}
& \text { (i) } Q_{ \pm}^{r}=0, \quad\left[\mathcal{H}_{v}, Q_{ \pm}\right]=0, \quad Q_{-}^{\dagger}=Q_{+} \\
& \text {(ii) } Q_{-}^{r-2} \mathcal{H}_{v}=Q_{-}^{r-1} Q_{+}+Q_{-}^{r-2} Q_{+} Q_{-}+\cdots+Q_{-} Q_{+} Q_{-}^{r-2}+Q_{+} Q_{-}^{r-1}
\end{aligned}
$$

Proof. From the commutation relation (7), we can show by induction that

$$
A_{+} A_{-}^{k}=A_{-}^{k} A_{+}-\vartheta_{k} A_{-}^{k-1}, \quad k \geq 1,
$$

where

$$
\vartheta_{k}= \begin{cases}k, & \text { if } k \text { is even, } \\ k+2 v R, & \text { if } k \text { is odd. }\end{cases}
$$

Combining this with Equation (20), we obtain, for, $k=1, \cdots, r-2$ :

$$
\begin{aligned}
Q_{+} Q_{-}^{r-1} & =A_{-}^{r-2}\left(A_{-} A_{+}-\vartheta_{r-1}\right) \Pi_{r-1} \\
Q_{-}^{r-1} Q_{+} & =A_{-}^{r-2} A_{-} A_{+} \Pi_{r-2} \\
Q_{-}^{r-1-k} Q_{+} Q_{-}^{k} & =A_{-}^{r-2}\left(A_{-} A_{+}-\vartheta_{k}\right)\left(\Pi_{r-2}+\Pi_{r-1}\right) .
\end{aligned}
$$

Additionally, a straightforward computation shows that

$$
\sum_{k=1}^{r-1} \vartheta_{k}=\frac{r(r-1)}{2}+2 v\left[\frac{r}{2}\right] R
$$

Thus, we get

$$
\begin{aligned}
\sum_{k=0}^{r-1} Q_{-}^{r-1-k} Q_{+} Q_{-}^{k} & =A_{-}^{r-2}\left[(r-1) A_{-} A_{+}\left(\Pi_{r-2}+\Pi_{r-1}\right)-\left(\sum_{k=1}^{r-2} \vartheta_{k}\right) \Pi_{r-2}-\left(\sum_{k=1}^{r-1} \vartheta_{k}\right) \Pi_{r-1}\right] \\
& =Q_{-}^{r-2}\left[(r-1) A_{-} A_{+}-\Theta_{1} \Pi_{r-2}-\Theta_{0} \Pi_{r-1}\right] .
\end{aligned}
$$


From Equation (13), we easily see that

$$
\left(\Pi_{r-2}+\Pi_{r-1}\right) \sum_{k=2}^{r-1} \Theta_{k} \Pi_{r-k-1}=0
$$

and combining with Equation (23), we get

$$
\sum_{k=0}^{r-1} Q_{-}^{r-1-k} Q_{+} Q_{-}^{k}=Q_{-}^{r-2} \mathcal{H}_{v}
$$

It remains to prove that $\left[\mathcal{H}_{v}, Q_{-}\right]=\left[\mathcal{H}_{v}, Q_{+}\right]=0$. Observe that for $k=0, \cdots, r-1$, we have

$$
r-1=\left[\frac{2 r+(-1)^{k}-1}{4}\right]+\left[\frac{2 r+(-1)^{k+1}-1}{4}\right],
$$

and then, for $k=0, \cdots, r-2$, we have

$$
\Theta_{k}-(r-1)(1-2 v R)=\Theta_{k+1}
$$

which leads to

$$
\begin{aligned}
Q_{-} \mathcal{H}_{v} & =\left\{(r-1) A_{-} A_{+}+1-2 v R-\sum_{k=0}^{r-2} \Theta_{k} \Pi_{r-k-2}\right\} A_{-}\left(1-\Pi_{0}\right) \\
& =\left\{(r-1) A_{-} A_{+}-\sum_{k=0}^{r-2} \Theta_{k+1} \Pi_{r-k-2}\right\}\left(1-\Pi_{r-1}\right) A_{-} \\
& =\mathcal{H}_{v} Q_{-} .
\end{aligned}
$$

Finally, we have obtained $\left[\mathcal{H}_{v}, Q_{-}\right]=0$, and since the operator $\mathcal{H}_{v}$ is self-adjoint and $Q_{+}=Q_{-}^{*}$, we conclude that $\left[\mathcal{H}_{v}, Q_{+}\right]=0$.

Proposition 2. For even integer $r$, the fractional supersymmetric Dunkl harmonic oscillator $\mathcal{H}_{v}$ has $r / 2-$ fold degenerate spectrum and acts on the wave functions $\psi_{n}^{(v)}(x)$ as:

$$
\mathcal{H}_{v} \psi_{n r+s}^{(v)}(x)=\lambda_{n r} \psi_{n r+s}^{(v)}(x), \quad s=0,1, \ldots r-1, \quad n=0,1,2, \ldots
$$

where

$$
\lambda_{n r}=(r-1)\left(n r+v+\frac{r+1}{2}\right)+(-1)^{s} v r, \quad s=0, \ldots, r-1 .
$$

Proof. From ([15] [formulas (3.7.1) and (3.7.2)]), the creation and annihilation operators $A_{+}$and $A_{-}$ act on the wave functions $\psi_{n r+s}^{v}$ as:

$$
\begin{aligned}
& A_{-} \psi_{n r+s}^{v}=\sqrt{n r+s+v\left(1-(-1)^{s}\right)} \psi_{n r+s-1}^{v} \\
& A_{+} \psi_{n r+s}^{v}=\sqrt{n r+s+1+v\left(1-(-1)^{s+1}\right)} \psi_{n r+s+1}^{v} .
\end{aligned}
$$

Then, the supercharges $Q_{-}$and $Q_{+}$take the value

$$
\begin{aligned}
& Q_{-} \psi_{n r+s}^{v}=\sqrt{\left(n r+s+v\left(1-(-1)^{s}\right)\right) / 2} \psi_{n r+s-1}^{v}, s=1, \cdots, r-1, \\
& Q_{+} \psi_{n r+s}^{v}=\sqrt{\left(n r+s+1+v\left(1-(-1)^{s+1}\right)\right) / 2} \psi_{n r+s+1}^{v}, s=0, \cdots, r-2, \\
& Q_{-} \psi_{n r}^{v}=0, \quad Q_{+} \psi_{(n+1) r-1}^{v}=0 .
\end{aligned}
$$


A straightforward computation shows that

$$
\mathcal{H}_{v} \psi_{n r+s}^{v}=\lambda_{n r} \psi_{n r+s}^{v}, \quad s=0, \cdots, r-1,
$$

where $\lambda_{n r}=(r-1)\left(n r+v+\frac{r+1}{2}\right)+(-1)^{s} v r$.

\section{Supersymmetric Generalized Hermite Polynomials}

\subsection{Associated Generalized Hermite Polynomials}

Starting form the following recurrence relations for the generalized Hermite polynomials $\left\{H_{n}^{(v)}(x)\right\}$,

$$
\begin{aligned}
& H_{n+1}^{(v)}(x)=2 x H_{n}^{(v)}(x)-2\left(n+v\left(1-(-1)^{n}\right)\right) H_{n-1}^{(v)}(x) \\
& H_{0}^{(v)}(x)=1, \quad H_{1}^{(v)}(x)=2 x
\end{aligned}
$$

given in [15-17], one defines, for each real number $c$, the system of polynomials $H_{n}^{(v)}(x, c)$ by the recurrence relation:

$$
H_{n+1}^{(v)}(x, c)=2 x H_{n}^{(v)}(x, c)-2\left(n+c+v\left(1-(-1)^{n}\right)\right) H_{n-1}^{(v)}(x, c),
$$

with initial conditions

$$
H_{0}^{(v)}(x, c)=1, \quad H_{1}^{(v)}(x, c)=2 x
$$

Now, assume that

$$
c>0, \quad c+2 v>-1 .
$$

By Favard's theorem [16], it follows that the family of polynomials $\left\{H_{n}^{(v)}(x, c)\right\}$ satisfying the recurrence relation (29) and the initial condition (30), is orthogonal with respect to some positive measure on the real line. We shall refer to the polynomials $\left\{H_{n}^{(v)}(x, c)\right\}$ as the associated generalized Hermite polynomials. As shown in ([18] Theorem 5.6.1)(see also [19-21]), there are two different systems of associated Laguerre polynomials denoted by $L_{n}^{(v)}(x, c)$ and $\mathcal{L}_{n}^{(v)}(x, c)$. They satisfy the recurrence relations:

$$
\begin{aligned}
& (2 n+2 c+v+1-x) L_{n}^{(v)}(x, c)=(n+c+1) L_{n+1}^{(v)}(x, c)+(n+c+v) L_{n-1}^{(v)}(x, c), \\
& L_{0}^{(v)}(x, c)=1, \quad L_{1}^{(v)}(x, c)=\frac{2 c+v+1-x}{c+1}
\end{aligned}
$$

and

$$
\begin{aligned}
& (2 n+2 c+v+1-x) \mathcal{L}_{n}^{(v)}(x, c)=(n+c+1) \mathcal{L}_{n+1}^{(v)}(x, c)+(n+c+v) \mathcal{L}_{n-1}^{(v)}(x, c) \\
& \mathcal{L}_{0}^{(v)}(x, c)=1, \quad \mathcal{L}_{1}^{(v)}(x, c)=\frac{c+v+1-x}{c+1}
\end{aligned}
$$

Recall the Tricomi function $\Psi(a, c ; x)$ given by

$$
\Psi(a, c ; x)=\frac{1}{\Gamma(a)} \int_{0}^{\infty} e^{-x t} t^{a-1}(1+t)^{c-a-1} d t, \quad \Re(a), \Re(x)>0 .
$$


By [18], the polynomials $L_{n}^{(v)}(x, c)$ and $\mathcal{L}_{n}^{(v)}(x, c)$ satisfy the orthogonality relations

$$
\begin{aligned}
\int_{0}^{\infty} L_{n}^{(v)}(x, c) L_{m}^{(v)}(x, c) x^{v} e^{-x} \frac{\left|\Psi\left(c, 1-v ; x e^{-i \pi}\right)\right|^{-2}}{\Gamma(c+1) \Gamma(v+c+1)} d x=\frac{(v+c+1)_{n}}{(c+1)_{n}} \delta_{n m}, \\
\int_{0}^{\infty} \mathcal{L}_{n}^{(v)}(x, c) \mathcal{L}_{m}^{(v)}(x, c) x^{v} e^{-x} \frac{\left|\Psi\left(c,-v ; x e^{-i \pi}\right)\right|^{-2}}{\Gamma(c+1) \Gamma(v+c+1)} d x=\frac{(v+c+1)_{n}}{(c+1)_{n}} \delta_{n m}
\end{aligned}
$$

when one of the following conditions is satisfied:

$$
v+c>-1, \quad c \geq 0 \quad \text { or } \quad v+c \geq-1, \quad c \geq-1 .
$$

The monic polynomial version of $H_{n}^{v}(x, c)$ is given by

$$
\mathcal{H}_{n}^{(v)}(x, c)=2^{-n} H_{n}^{(v)}(x, c), n=0,1, \cdots,
$$

and satisfies

$$
\begin{aligned}
& \mathcal{H}_{n+1}^{(v)}(x, c)=x \mathcal{H}_{n}^{(v)}(x, c)-\frac{1}{2}\left(n+c+v\left(1-(-1)^{n}\right)\right) \mathcal{H}_{n-1}^{(v)}(x, c), \\
& \mathcal{H}_{-1}^{(v)}(x, c)=0, \quad \mathcal{H}_{0}^{(v)}(x, c)=1 .
\end{aligned}
$$

It is easy to see that the polynomial $(-1)^{n} \mathcal{H}_{n}^{(v)}(-x, c)$ also satisfies (38). Thus,

$$
\mathcal{H}_{n}^{(v)}(-x, c)=(-1)^{n} \mathcal{H}_{n}^{(v)}(x, c) .
$$

Thus, by induction, we write them in the form

$$
\mathcal{H}_{2 n}^{(v)}(x, c)=S_{n}\left(x^{2}\right) \quad \text { and } \quad \mathcal{H}_{2 n+1}^{(v)}(x, c)=x Q_{n}\left(x^{2}\right),
$$

where $S_{n}(x), Q_{n}(x)$ are monic polynomials of degree $n$.

Theorem 2. Let $c>0$ and $v>-c / 2$. The associated generalized Hermite polynomials $H_{n}^{(v)}(x, c)$, defined in (29), have the explicit form:

$$
\begin{aligned}
& H_{2 n}^{(v)}(x, c)=(-1)^{n} 2^{2 n}(1+c / 2)_{n} \mathcal{L}_{n}^{(v-1 / 2)}\left(x^{2}, c / 2\right), \\
& H_{2 n+1}^{(v)}(x, c)=(-1)^{n} 2^{2 n+1}(1+c / 2)_{n} x L_{n}^{(v+1 / 2)}\left(x^{2}, c / 2\right),
\end{aligned}
$$

and the orthogonality relations

$$
\int_{\mathbb{R}} H_{n}^{(v)}(x, c) H_{m}^{(v)}(x, c)|x|^{2 v} e^{-x^{2}} \frac{\left|\Psi\left(c / 2,1 / 2-v ; x^{2} e^{-i \pi}\right)\right|^{-2}}{\Gamma(1+c / 2) \Gamma(v+c / 2+1 / 2)}=\zeta_{n} \delta_{n m},
$$

where

$$
\zeta_{n}=\left\{\begin{array}{lll}
2^{4 k}(1+c / 2)_{k}(v+c / 2+1 / 2)_{k}, & \text { if } & n=2 k \\
2^{4 k+2}(1+c / 2)_{k}(v+c / 2+3 / 2)_{k}, & \text { if } & n=2 k+1 .
\end{array}\right.
$$


Proof. It is directly verified that the polynomials $S_{n}(x), Q_{n}(x)$ given in (39) are orthogonal as they satisfy the recurrence relations

$$
\begin{aligned}
S_{n+1}(x)= & (x-(2 n+c+v+1 / 2)) S_{n}(x)-(n+c / 2) \\
& \times(n+c / 2-1 / 2+v) S_{n-1}(x), \\
S_{-1}(x)= & 0, \quad S_{0}(x)=1,
\end{aligned}
$$

and

$$
\begin{aligned}
Q_{n+1}(x)= & \left.(x-(2 n+c+3 / 2+v)) Q_{n}(x)-(n+c / 2) b i g\right) \\
& \times(n+(1+c) / 2+v) Q_{n-1}(x) \\
Q_{-1}(x)= & 0, \quad Q_{0}(x)=1 .
\end{aligned}
$$

From Equation (32), we see that the polynomials $S_{n}(x)$ satisfy the same recurrence relation as $(-1)^{n}(1+$ $c / 2)_{n} \mathcal{L}_{n}^{(v-1 / 2)}(x, c / 2)$, so that

$$
S_{n}(x)=(-1)^{n}(1+c / 2)_{n} \mathcal{L}_{n}^{(v-1 / 2)}(x, c / 2) .
$$

A similar analysis shows that

$$
Q_{n}(x)=(-1)^{n}(1+c / 2)_{n} L_{n}^{(v+1 / 2)}(x, c / 2) .
$$

In view of Equations (41) and (42), the explicit form of the associated generalized Hermite polynomials is given by

$$
\begin{aligned}
& H_{2 n}^{(v)}(x, c)=(-1)^{n} 2^{2 n}(1+c / 2)_{n} \mathcal{L}_{n}^{(v-1 / 2)}\left(x^{2}, c / 2\right), \\
& H_{2 n+1}^{(v)}(x, c)=(-1)^{n} 2^{2 n+1}(1+c / 2)_{n} x L_{n}^{(v+1 / 2)}\left(x^{2}, c / 2\right) .
\end{aligned}
$$

From Equations (36) and (37), we deduce that the system $\mathcal{H}_{n}^{v}(x, c)$ satisfies the orthogonality relations

$$
\int_{\mathbb{R}} H_{n}^{(v)}(x, c) H_{m}^{(v)}(x, c)|x|^{2 v} e^{-x^{2}} \frac{\left|\Psi\left(c / 2,1 / 2-v ; x^{2} e^{-i \pi}\right)\right|^{-2}}{\Gamma(1+c / 2) \Gamma(v+c / 2+1 / 2)}=\zeta_{n} \delta_{n m}
$$

with

$$
\zeta_{n}=\left\{\begin{array}{lll}
2^{4 k}(1+c / 2)_{k}(v+c / 2+1 / 2)_{k}, & \text { if } & n=2 k \\
2^{4 k+2}(1+c / 2)_{k}(v+c / 2+3 / 2)_{k}, & \text { if } & n=2 k+1
\end{array}\right.
$$

\subsection{Supersymmetric Generalized Hermite Polynomials}

In the sequel, we assume that $r$ is an even integer and we consider the Hermitian supercharge operator $Q$, defined on $\mathcal{S}(\mathbb{R})$, by

$$
Q=\frac{1}{\sqrt{2}} Y_{v}\left(\Pi_{r-1}-\Pi_{0}\right)+\frac{x}{\sqrt{2}}\left(2-\Pi_{0}-\Pi_{r-1}\right) .
$$

From Equation (14), we have

$$
Q=\frac{1}{\sqrt{2}}\left(Q_{-}+Q_{+}\right)
$$


so it has a self-adjoint extension on $L^{2}\left(\mathbb{R},|x|^{2 v} d x\right)$. Furthermore, it acts on the basis $\psi_{n}^{v}$ as

$$
\begin{aligned}
& Q \psi_{n r+s}^{v}=a_{s}^{(n)} \psi_{n r+s-1}^{v}+a_{s+1}^{(n)} \psi_{n r+s+1}^{v}, s=1, \cdots, r-1, \\
& Q \psi_{n r}^{v}=a_{1}^{(n)} \psi_{n r+1}^{v}, \quad Q \psi_{(n+1) r-1}^{v}=a_{r-1}^{(n)} \psi_{(n+1) r-1}^{v},
\end{aligned}
$$

where

$$
a_{s}^{(n)}:=\sqrt{\left(n r+s+v\left(1-(-1)^{s}\right)\right) / 2}, \quad s=1, \cdots, r-1 .
$$

On the other hand, by (46), we see that the operator $Q$ leaves invariant the finite dimensional subspace of $L^{2}\left(\mathbb{R},|x|^{2 v} d x\right)$ generated by $\psi_{n r+s}^{v}, s=0,1, \cdots, r-1$. Hence, $Q$ can be represented in this basis by the following $r \times r$ tridiagonal Jacobi matrix $A_{r}^{(n)}$

$$
A_{r}^{(n)}=\left(\begin{array}{cccccc}
0 & a_{1}^{(n)} & 0 & & & \\
a_{1}^{(n)} & 0 & a_{2}^{(n)} & 0 & & \\
0 & a_{2}^{(n)} & 0 & a_{3}^{(n)} & \ddots & \\
& \ddots & \ddots & \ddots & \ddots & 0 \\
& & \ddots & a_{r-2}^{(n)} & 0 & a_{r-1}^{(n)} \\
& & & 0 & a_{r-1}^{(n)} & 0
\end{array}\right) .
$$

It is well known that, if the coefficients of the subdiagonal of some Jacobi Matrix are different from zero, then all the eigenvalues of this matrix are real and nondegenerate [16]. We introduce the normalized eigenvectors $\phi_{s}$ of the supercharge $Q$

$$
Q \phi_{s}=x_{s} \phi_{s}, \quad s=0, \cdots, r-1
$$

that can be expanded in the basis $\psi_{n r+k}, k=0,1, \cdots, r-1$, as

$$
\phi_{s}=\sum_{k=0}^{r-1} \sqrt{w_{s}} p_{k}\left(x_{s}\right) \psi_{n r+k}
$$

where the coefficients $p_{k}$ obey the three-term recurrence relation [22]

$$
\begin{aligned}
& a_{k}^{(n)} p_{k-1}(x)+a_{k+1}^{(n)} p_{k+1}(x)=x p_{k}(x) \\
& p_{-1}(x)=0, \quad p_{0}\left(x_{s}\right)=1
\end{aligned}
$$

Hence, they become orthogonal polynomials. We denote by $P_{k}(x)$, the monic orthogonal polynomial related to $p_{k}(x)$ by

$$
P_{k}(x)=h_{k} p_{k}(x)
$$

where

$$
h_{k}=a_{k}^{(n)} \cdots a_{1}^{(n)}
$$

and satisfying

$$
\begin{aligned}
& x P_{k}(x)=P_{k+1}(x)+\frac{1}{2}\left(k+n r+v\left(1-(-1)^{k}\right)\right) P_{k-1}(x), k=0, \cdots, r-1, \\
& P_{-1}(x)=0, \quad P_{0}(x)=1 .
\end{aligned}
$$


From the three terms recurrence relations (51), the polynomials $P_{k}(x)$ can be identified with the associated generalized Hermite polynomial $\mathcal{H}_{k}^{(v)}(x, c)$, namely,

$$
P_{k}(x)=\mathcal{H}_{k}^{(v)}(x, n r)
$$

It is well known from the theory of orthogonal polynomials that the eigenvalues of the Jacobi matrix $A_{r}^{(n)}$ coincide with the roots of the characteristic polynomial $\mathcal{H}_{r}^{(v)}(x, n r)[16,22]$. The weights $w_{s}$ defined in (56) are given by the following formula

$$
w_{s}=\frac{h_{r}^{2}}{\mathcal{H}_{r-1}^{(v)}\left(x_{s}, n r\right)\left(\mathcal{H}_{r}^{(v)}\right)^{\prime}\left(x_{s}, n r\right)},
$$

where $\left(\mathcal{H}_{r}^{(v)}\right)^{\prime}(x, n r)$ denotes the derivative of $\mathcal{H}_{r}^{(v)}(x, n r), h_{r}$ is defined in Equation (50) and $x_{n r, 1}>$ $\cdots>x_{n r, r}$ are the zeros of $\mathcal{H}_{r}^{(v)}(x, n r)$. For more detail, we refer to [16]. Then, it turns out that

$$
\phi_{s}=\sum_{k=0}^{r-1} u_{k s}^{(n)} \psi_{n r+k}
$$

where

$$
u_{k s}^{(n)}=\frac{h_{r}}{h_{k}} \frac{\mathcal{H}_{k}^{(v)}\left(x_{s}, n r\right)}{\left(\mathcal{H}_{r-1}^{(v)}\left(x_{s}, n r\right)\left(\mathcal{H}_{r}^{(v)}\right)^{\prime}\left(x_{s}, n r\right)\right)^{1 / 2}}, \quad 0 \leq s, k \leq r-1 .
$$

Since both bases $\left\{\psi_{n r+k}, k=0, \cdots r-1\right\}$ and $\left\{\phi_{s}, s=0, \cdots r-1\right\}$ are orthonormal and all the coefficients are real, then the matrix $\left(u_{k s}^{(n)}\right)$ is orthogonal and hence the system $\left\{\mathcal{H}_{k}^{(v)}(x)\right\}$ becomes orthogonal polynomials:

$$
\sum_{s=0}^{r-1} w_{s} \mathcal{H}_{k}^{(v)}\left(x_{s}\right) \mathcal{H}_{k^{\prime}}^{(v)}\left(x_{s}\right)=\delta_{k k^{\prime}} / h_{k}^{2}
$$

We call supersymmetric generalized Hermite polynomials the orthogonal polynomials, denoted by $\mathbb{H}_{N}^{(r, v)}(x)$, extracted form the orthogonal function $\phi_{s}$ :

$$
\mathbb{H}_{N}^{(r, v)}(x)=\sum_{k=0}^{r-1} H_{k}^{(v)}\left(x_{s}, n r\right) H_{n r+k}^{(v)}(x), N=n r+s,
$$

and we obtain the following:

Theorem 3. The supersymmetric generalized Hermite polynomials $\mathbb{H}_{N}^{(r, v)}(x)$ satisfy the orthogonality relations

$$
\int_{-\infty}^{\infty} \mathbb{H}_{N}^{(r, v)}(x) \mathbb{H}_{N^{\prime}}^{(r, v)}(x)|x|^{2 v} e^{-x^{2}} d x=\varrho_{N} \delta_{N N^{\prime}}
$$

where $\varrho_{N}=\gamma_{n r} / w_{s}$ for $s=0, \cdots r-1$ and $N=n r+s$.

Proof. From Equations (10) and (56), we obtain

$$
\begin{aligned}
\int_{-\infty}^{\infty} \mathbb{H}_{n r+s}^{(r, v)}(x) \mathbb{H}_{n^{\prime} r+s^{\prime}}^{(r, v)}(x)|x|^{2 v} e^{-x^{2}} d x & =\delta_{n n^{\prime}} \sum_{k=0}^{r-1} H_{k}^{(v)}\left(x_{s}, n r\right) H_{k}^{(v)}\left(x_{s^{\prime}}, n r\right) \gamma_{n r+k} \\
& =\delta_{n n^{\prime}} \gamma_{n r} \sum_{k=0}^{r-1} h_{k}^{2} \mathcal{H}_{k}^{(v)}\left(x_{s}, n r\right) \mathcal{H}_{k}^{(v)}\left(x_{s^{\prime}}, n r\right)
\end{aligned}
$$


and, from ([18] Theorem 2.11.2), we obtain the dual orthogonality relation for $\left\{\mathcal{H}_{k}^{(v)}(x)\right\}$ :

$$
\sum_{k=0}^{r-1} \mathcal{H}_{k}^{(v)}\left(x_{s}\right) \mathcal{H}_{k}^{(v)}\left(x_{s^{\prime}}\right) \frac{\left[\frac{n r+k}{2}\right] ! \Gamma\left(\frac{n r+k+1}{2}+v+\frac{1}{2}\right)}{\left[\frac{n r}{2}\right] ! \Gamma\left(\frac{n r+1}{2}+v+\frac{1}{2}\right)}=\delta_{s s^{\prime}} / w_{s}
$$

and, finally,

$$
\int_{-\infty}^{\infty} \mathbb{H}_{n r+s}^{(r, v)}(x) \mathbb{H}_{n^{\prime} r+s^{\prime}}^{(r, v)}(x)|x|^{2 v} e^{-x^{2}} d x=\delta_{n n^{\prime}} \delta_{s s^{\prime}} \gamma_{n r} / w_{s}
$$

Author Contributions: Formal analysis, F.B. and W.J.; Methodology, F.B. and W.J.; Writing-original draft, F.B. and W.J.; Writing-review and editing, F.B. and W.J. All authors have read and agreed to the published version of the manuscript.

Funding: The authors would like to extend their sincere appreciation to the Deanship of Scientific Research at King Saudi University for funding this Research Group No. (RG-1437-020).

Conflicts of Interest: The authors declare no conflict of interest.

\section{References}

1. Plyushchay, M.S. Deformed Heisenberg algebra, fractional spin fields and supersymmetry without fermions Annals Phys. 1996, 245, 339-360.

2. Witten, E. Dynamical breaking of supersymmetry. Nucl. Phys. B 1981, 188, 513-554 [CrossRef]

3. Daoud, M.; Hassouni, Y.; Kibler, M. The k-fermions as objects interpolating between fermions and bosons. In Symmetries in Science X; Daoud, M., Hassouni, Y., Kibler, M., Gruber, B., Ramek, M., Eds.; Plenum Press: New York, NY, USA, 1998; pp. 63-77.

4. Plyushchay, M.S. Supersymmetry without fermions DFTUZ-94-05. arXiv 1994, arXiv:hep-th/9404081.

5. Post, S.; Vinet, L.; Zhedanov, A. Supersymmetric quantum mechanics with reflections. J. Phys. A Math. Theor. 2011, 44, 435301. [CrossRef]

6. Cooper, F.; Khare, A.; Sukhatme, U. Supersymmetry in Quantum Mechanics; World Scientific: Singapore, 2001

7. Khare, A. Parasupersymmetric quantum mechanics of arbitrary order. J. Phys. A 1992, 25, L749. [CrossRef]

8. Quesne, C.; Vansteenkiste, N. $\mathrm{C}_{\lambda}$-extended harmonic oscillator and (para) supersymmetric quantum mechanics. Phys. Lett. A 1998, 240, 21. [CrossRef]

9. Plyushchay, M. Hidden nonlinear supersymmetries in pure parabosonic systems, Int. J. Mod. Phys. A 2000, 15, 3679-3698. [CrossRef]

10. Plyushchay, M.S. Deformed Heisenberg algebra with reflection. Nuclear Phys. B 1997, 491, 619-634. [CrossRef]

11. Ghazouani, S.; Bouzeffour, F. A fractional power for Dunkl transform. Bull. Math. Anal. Appl. 2014, 6, 1-30.

12. Ghazouani, S.; Bouzeffour, F. Heisenberg uncertainty principle for a fractional power of the Dunkl transform on the real line. J. Computat. Appl. Math. 2016, 294, 151-176. [CrossRef]

13. Watson, G.N. A Treatise on the Theory of Bessel Functions, 2nd ed.; Cambridge Univ. Press: London, UK; New York, NY, USA, 1944.

14. Dunkl, C.F.; Xu, Y. Orthogonal polynomials of several variables. In Encyclopedia of Mathematics and Its Applications; Cambridge University Press: Cambridge, UK, 2001; Volume 81.

15. Rosenblum, M. Generalized Hermite polynomials and the Bose-like oscillator calculus. In Non Self-Adjoint Operators and Related Topics; Sheva, B., Ed.; Springer Basel AG: Birkhauser Verlag: Basel, Switzerland, 1994.

16. Chihara, T. An Introduction to Orthogonal Polynomials. In Dover Books on Mathematics, Reprint ed.; Gordon and Breach: New York, NY, USA, 2011.

17. Szegö, G. Orthogonal Polynomials; American Mathematical Society: Providence, RI, USA, 1939; Volume 23.

18. Ismail, M.E.H. Classical and quantum orthogonal polynomials in one variable. In Encyclopedia of Mathematics and its Applications; Cambridge University Press: Cambridge, UK. 2005; ISBN 0521782015.

19. Askey, R.; Wimp, J. Associated Laguerre and Hermite polynomials. Proc. Roy. Soc. Edinburgh Sect. A 1984, 96, 15-37. [CrossRef] 
20. Kim, T.; Kim, D.; Jang, L.-C.; Kwon, H. On differential equations associated with squared hermite polynomials. J. Comput. Anal. Appl. 2017, 23, 1252-1264.

21. Kim, T.; Kim, D.S.; Dolgy, D.V.; Park, J.-W. Sums of finite products of Legendre and Laguerre polynomials. Adv. Differ. Equ. 2018, 2018, 277. [CrossRef]

22. Atkinson, F.V. Discrete and Continuous Boundary Problems; Academic Press: New York, NY, USA; London, UK, 1964.

(C) 2020 by the authors. Licensee MDPI, Basel, Switzerland. This article is an open access article distributed under the terms and conditions of the Creative Commons Attribution (CC BY) license (http://creativecommons.org/licenses/by/4.0/). 